\title{
Diabetic retinopathy: looking beyond the eyes
}

\author{
Rafael Simó $^{1,2}$ (D) Coen D. A. Stehouwer ${ }^{3,4} \cdot$ Angelo Avogaro $^{5}$
}

Received: 21 April 2020 / Accepted: 8 May 2020 / Published online: 18 June 2020

(C) Springer-Verlag GmbH Germany, part of Springer Nature 2020

Keywords Cardiovascular disease $\cdot$ Cognitive decline $\cdot$ Dementia $\cdot$ Diabetic microangiopathy $\cdot$ Diabetic retinopathy Neurodegeneration

\author{
Abbreviation \\ CVD Cardiovascular disease
}

To the Editor: The eye as a tool for identifying systemic diseases has fascinated scientists for centuries. In the era of precision medicine, screening for diabetic retinopathy has emerged as a renewed cost-effective method, not only to prevent sight-threatening disease, but also to identify patients more prone to develop other diabetic complications $[1,2]$. In recent years, evidence has accumulated indicating that diabetic retinopathy is not only a herald of microvascular complications, but also of macrovascular complications, such as ischaemic coronary disease and stroke [2,3]. In addition, the presence of diabetic retinopathy is an independent risk factor for cognitive impairment and dementia and, consequently, is a useful tool to identify candidates for the screening of cognitive status [4].

The current EASD/ADA guideline for treating type 2 diabetes relies on the presence of cardiovascular disease (CVD), but there is no mention of how the presence of

Rafael Simó

rafael.simo@vhir.org

1 Diabetes and Metabolism Research Unit, Vall d'Hebron Research Institute, Vall d'Hebron University Hospital, Pg. Vall d'Hebron 119 129, 08035 Barcelona, Spain

2 Centro de Investigación Biomédica en Red de Diabetes y Enfermedades Metabólicas Asociadas, Instituto de Salud Carlos III, Madrid, Spain

3 Department of Internal Medicine, Maastricht University Medical Centre, Maastricht, the Netherlands

4 Cardiovascular Research Institute Maastricht, Maastricht University Medical Centre, Maastricht, the Netherlands

5 Section of Diabetes and Metabolic Diseases, Department of Medicine, University of Padova, Padova, Italy diabetic retinopathy can modulate the current management of the disease [5]. In this comment, several important points related to this commonly forgotten issue will be underlined.

\section{Diabetic retinopathy and CVD}

There is epidemiological evidence showing that either diabetic retinopathy or diabetic macular oedema (DMO) independently predict incident coronary heart disease, fatal CVD, heart failure and stroke $[2,3]$. In addition, the presence and degree of diabetic retinopathy has recently been found to be an independent and powerful predictor of subclinical CVD [6]. Therefore, screening for CVD should be prioritised in patients with diabetic retinopathy.

Although the underlying molecular mechanisms linking diabetic retinopathy and CVD are still a matter of debate, there are notable similarities in their pathophysiology. In this regard, recent evidence indicates that, in individuals with type 2 diabetes, the vasa vasorum (a network of small blood vessels that supply the walls of large blood vessels) present evolutionary changes similar to those observed in the retina: an initial stage in which endothelial dysfunction and loss of capillaries predominate [7], and more advanced stages in which ischaemia plays a key role, leading to angiogenesis and inflammation in response to the progressive enlargement of the necrotic core within the plaque [8]. This change in plaque phenotype results in a more inflamed and unstable plaque, favouring plaque rupture and a poor outcome of cardiovascular events. Maladaptive vascular reparatory mechanisms may also play a role; a decrease in the number of endothelial colony forming cells (ECFCs) not only predict macroangiopathy but also diabetic retinopathy [9]. Despite these similarities, the vascular beds in the blood vessels in the heart show important differences to those in the retina that could affect the response to treatment approaches. For instance, anti-vascularendothelial-growth-factor (VEGF) agents are effective for 
treating proliferative diabetic retinopathy but exert a deleterious effect in coronary arteries by inhibiting the revascularisation after ischaemia [10].

The main structural retinal fingerprints associated with CVD and stroke are displayed in Fig. 1. The presence of hard exudates (a component of non-proliferative diabetic retinopathy) has been particularly associated with CVD and plaque formation $[3,11]$. In fact, hard exudates in the retina and plaques in large arteries are the result of leakage of lipids and proteinaceous material through the endothelial barrier, and this may explain why they are related.

In addition, impairment of retinal neurovascular coupling, as assessed by flicker-induced arterial dilatation, is more frequent in patients with coronary artery disease, and it is even more pronounced in patients with heart failure [12]. Notably, the impairment of the neurovascular unit is an early functional abnormality in diabetes that can be detected even before structural changes.

\section{Diabetic retinopathy and cognitive impairment}

Individuals with type 2 diabetes have a higher risk of developing cognitive dysfunction and neurodegenerative diseases than age-matched non-diabetic individuals [4]. Since the retina is a brain-derived tissue, it has been suggested that the study of the retina could give valuable information regarding the status of the brain. In fact, retinal neurodegeneration is an early event in the pathogenesis of diabetic retinopathy, and several pathways involved in neurodegenerative diseases, such as Alzheimer's and Parkinson's disease, have been found to be altered in the retinas from diabetic individuals [13]. Besides neurodegeneration, microvascular abnormalities of the retina in the setting of diabetic retinopathy have been related, not only to a high risk of stroke, but also to cognitive decline and dementia [4, 14]. Overall, these associations may be explained by viewing diabetes as a slowly progressive neuro-microvascular disorder that affects the retina and the brain in parallel and that begins at a very early stage of the disease. The retinal fingerprints that should alert the clinicians of an increased risk of cognitive impairment are shown in Fig. 1.

The value of retinal structural imaging and its functional assessments as predictors of cognitive impairment or dementia is currently evaluated in the RECOGNISED study (ClinicalTrials.gov registration no. NCT04281186). The hypothesis is that individuals with type 2 diabetes in whom neurodegeneration is a predominant event in retinal examinations will be more prone to develop rapid cognitive decline (as is the case in Alzheimer's disease) (Fig. 1). By contrast, in those individuals in whom retinal neurodegeneration is apparently absent, slow cognitive decline will occur and this will be mainly modulated by microvascular impairment.

\section{Scientific and clinical consequences}

Several take-home messages emerge from recent evidence linking micro- and macroangiopathy. Perhaps the most



Fig. 1 Main retinal findings associated with CVD, stroke and cognitive impairment. Retinal abnormalities linked to CVD and stroke are within the blue box. Retinal changes related to cognitive impairment comprise both the blue (vascular abnormalities) and the peach box (retinal neurodegeneration). When neurodegeneration is added to vascular impairment, the cognitive decline will be more pronounced. The retinal branching pattern can be quantified using several global parameters, including fractal dimension, tortuosity and branching angle. Angio-OCT, Optical coherence tomography angiography; NPDR, non-proliferative diabetic retinopathy; OCT, optical coherence tomography; PDR, proliferative diabetic retinopathy 
important is that diabetic retinopathy and CVD should no longer be considered as entirely separate entities in the setting of diabetes. In fact, diabetic retinopathy should be taken into account as a primary cardiovascular risk factor, at least on the same level as dyslipidaemia and hypertension $[3,6,15]$. The vast majority of studies on CVD in diabetic participants have not considered this important variable in the randomisation process and, therefore, this is a significant drawback that could bias the interpretation of results. Another important point is that the presence of diabetic retinopathy permits us to identify a subset of the diabetic population in which the screening for CVD should be prioritised and risk-factor control optimised.

Additionally, the possibility of identifying patients with type 2 diabetes who have cognitive impairment and prodromal stages of dementia will be crucial in planning treatment, thus allowing for a reduction in the frequency of severe hypoglycaemia and hospital admissions that this subset of the diabetic population presents with.

Finally, it should be noted that although there are fewer data on those with type 1 diabetes than with type 2 diabetes, it is assumed that the retinal findings associated with either CVD outcomes or dementia are generally similar.

\section{Conclusion}

In conclusion, the strategy of using retinal assessment, not only to prevent sight-threatening disease in diabetes, but also to identify patients at risk of CVD and dementia, opens up a new era in the management of individuals with diabetes.

Authors' relationships and activities The authors declare that there are no relationships or activities that might bias, or be perceived to bias, their work.

Contribution statement All authors were responsible for drafting the article and revising it critically for important intellectual content. All authors approved the version to be published.

\section{References}

1. Vujosevic S, Aldington SJ, Silva P et al (2020) Screening for diabetic retinopathy: new perspectives and challenges. Lancet Diabetes Endocrinol 8:337-347
2. Pearce I, Simó R, Lövestam-Adrian M, Wong DT, Evans M (2019) Association between diabetic eye disease and other complications of diabetes: implications for care. A systematic review. Diabetes Obes Metab 21:467-478

3. Xie J, Ikram MK, Cotch MF et al (2017) Association of diabetic macular edema and proliferative diabetic retinopathy with cardiovascular disease: a systematic review and meta-analysis. JAMA Ophthalmol 135(6):586-593

4. Simó R, Ciudin A, Simó-Servat O, Hernández C (2017) Cognitive impairment and dementia: a new emerging complication of type 2 diabetes - the diabetologist's perspective. Acta Diabetol 54(5): 417-424

5. Buse JB, Wexler DJ, Tsapas A et al (2020) 2019 update to: management of hyperglycaemia in type 2 diabetes, 2018. A consensus report by the American Diabetes Association (ADA) and the European Association for the Study of Diabetes (EASD). Diabetologia 63:221-228

6. Simó R, Bañeras J, Hernández C et al (2019) Diabetic retinopathy as an independent predictor of subclinical cardiovascular disease: baseline results of the PRECISED study. BMJ Open Diabetes Res Care 7:e000845

7. Gerstein HC, Nair V, Chaube R et al (2019) Dysglycemia and the density of the coronary vasa vasorum. Diabetes Care 42:980-982

8. Sedding DG, Boyle EC, Demandt JAF et al (2018) Vasa vasorum angiogenesis: key player in the initiation and progression of atherosclerosis and potential target for the treatment of cardiovascular disease. Front Immunol 9:706

9. Fadini GP, Albiero M, Bonora BM, Avogaro A (2019) Angiogenic abnormalities in diabetes mellitus: mechanistic and clinical aspects. J Clin Endocrinol Metab 104(11):5431-5444

10. Simó R, Hernández C (2008) Intravitreous anti-VEGF for diabetic retinopathy: hopes and fears for a new therapeutic strategy. Diabetologia 51:1574-1580

11. Klein R, Sharret AR, Klein BEK et al (2002) The associations of atherosclerosis, vascular risks factors, and retinopathy in adults with diabetes: the Atherosclerosis Risk in Communities Study. Ophthalmology 109:1225-1234

12. Barthelmes J, Nägele MP, Cantatore $\mathrm{S}$ et al (2019) Retinal microvascular dysfunction in patients with coronary artery disease with and without heart failure: a continuum? Eur J Heart Fail 21:988997

13. Sundstrom JM, Hernández C, Weber SR et al (2018) Proteomic analysis of early diabetic retinopathy reveals mediators of neurodegenerative brain diseases. Invest Ophthalmol Vis Sci 59:2264-2227

14. van Sloten TT, Sedaghat S, Carnethon MR, Launer LJ, Stehouwer CDA (2020) Cerebral microvascular complications of type 2 diabetes: stroke, cognitive dysfunction, and depression. Lancet Diabetes Endocrinol 8(4):325-336

15. Cheung N, Wang JJ, Klein R, Couper DJ, Sharret AR, Wong TY (2007) Diabetic retinopathy and the risk of coronary heart disease. Diabetes Care 30:1742-1746

Publisher's note Springer Nature remains neutral with regard to jurisdictional claims in published maps and institutional affiliations. 